\title{
A survey of Singapore anaesthesiologists for practice and prevention of peri-operative hypothermia in adult surgical patients
}

\section{Dear Editor,}

Core temperature is the temperature of blood and internal organs; influenced by biorhythm, metabolism, activity and hormones. It is regulated within a narrow range, ${ }^{1}$ but this is impaired during general and/or neuraxial anaesthesia. Inadvertent peri-operative hypothermia is defined as a core temperature of $<36^{\circ} \mathrm{C}$, the prevalence of which can be as high as $90 \% .^{2}$ This may lead to increased peri-operative complications (ranging from surgical site infection, pressure sores, cardiac morbidity, bleeding and delayed drug metabolism leading to longer hospitalisation and increased healthcare costs), patient discomfort and distress. ${ }^{3-5}$

The aims of this national survey and recommendations are to improve the safety of anaesthesia relating to the detection, prevention and treatment of inadvertent peri-operative hypothermia in adult patients. These recommendations are considered good practices and are not mandatory. Healthcare professionals should exercise discretion and make decisions for the individual patient.

Guidelines from Germany, ${ }^{5} \mathrm{US},{ }^{6} \mathrm{UK}^{7}$ and $\mathrm{Canada}^{8}$ were reviewed for the purpose of crafting recommendations for the College of Anaesthesiologists (CAS), Academy of Medicine, Singapore. The authors also used PubMed, Ovid and Google Scholar to search for terms such as "hypothermia", "perioperative", "intraoperative", "warming" and "temperature" in recent studies published in English.

A multi-hospital survey in Singapore involving 178 anaesthesiologists was performed in 2018 with ethics approval (DSRB: 2017/00973) and waiver of consent by the participants. Survey data are presented under appropriate headings in these recommendations. Opinions and feedback were sought from CAS members and Asian Anaesthesiology College Presidents at the CAS Refresher Course in Hanoi, Vietnam (6-7 April 2019). This document has been endorsed by the CAS Council in 2019.

Methods of peri-operative temperature measurement. Temperature is 1 of the 5 vital signs according to the Joint Commission. Under general anaesthesia, 47\% of the respondents "often" measured core temperature, compared to only $14 \%$ and $17 \%$ under neuraxial anaesthesia and peripheral nerve block respectively. Tympanic (81\%) and sublingual (13\%) were the commonest methods of temperature measurements preoperatively and post-operatively, with nasopharyngeal temperature monitoring being favoured (59\%) by anaesthesiologists in Singapore intra-operatively.

A survey in Europe reported that temperature monitoring was not appropriately performed in $>80 \%$ of patients. ${ }^{9}$ The temperature monitoring method should be based on the requirements of the procedure (anaesthesia type, accessibility and invasiveness of the route, etc.), and performed at monitoring sites with high homogeneous blood perfusion. Peripheral thermometers (temporal artery, axillary or oral) have been shown to have suboptimal clinical accuracy when compared to central thermometers (pulmonary artery catheter, urinary bladder, oesophageal) - especially among patients with fever and hypothermia. ${ }^{10}$

In the National Institute for Health and Care Excellence (NICE, UK) guidelines, direct measurement/ estimate of core temperature has been advocated to measure patients' temperature peri-operatively. ${ }^{7}$ This is the reading produced by a thermometer with no correction factor applied. The sites advocated include the distal oesophagus, urinary bladder, deep forehead (for zero heat-flux thermometry), rectum, sublingual and axilla. ${ }^{7}$ These recommendations do not advocate using indirect estimates of core temperature in peri-operative patients, where a correction factor is applied to the thermometer reading (e.g. infrared temporal/forehead, forehead strips). ${ }^{7}$

Infrared thermal radiation from the tympanic membrane is typically used peri-operatively because of its speed, ease of use, and low cost. To get best measurement results, the device has to be directed towards the tympanic membrane by straightening the cartilaginous part of the external ear. Contingent on practicality and availability of monitoring equipment in Singapore, we recommend tympanic, nasopharyngeal, oropharyngeal, sublingual, oesophageal, rectal and zero heat-flux techniques to be used for temperature measurement during the peri-operative period (Table 1).

Risk factors. In Singapore, less anaesthesiologists monitor patients' temperature (68\%) and conduct active warming for patients (90\%) undergoing thoracoscopic/ laparoscopic surgeries as opposed to patients undergoing open cavity surgery ( $87 \%$ and $93 \%$ respectively). Ninety-two percent of survey participants considered 


\section{General recommendations}

- All patients should be considered at risk of inadvertent peri-operative hypothermia. Special attention should be paid when there are multiple known risk factors present.

Risk factors include:

- American Society of Anesthesiologists (ASA) physical status score $\geq \mathrm{II}$

- Pre-operative temperature $<36^{\circ} \mathrm{C}$

- Receiving both general anaesthesia and neuraxial block

- Undergoing intermediate and major surgery

- Surgical duration $>30$ minutes

- Older patients (e.g. $>65$ years)

- Poor nutritional status / low body weight

- Significant comorbidities (e.g. diabetes mellitus, hypothyroidism, trauma, burns, cardiovascular disease)

- We recommend tympanic, nasopharyngeal, oropharyngeal, sublingual, oesophageal, rectal and zero heat-flux techniques to be used for temperature measurement during the peri-operative period.

\section{Pre-operative period}

- The temperature of the patient should to be taken and documented prior to anaesthesia. If the temperature is $<36.0^{\circ} \mathrm{C}$, active warming methods may be undertaken.

- Active pre-warming 10-30 minutes prior to anaesthesia may be considered good practice especially in patients with multiple known risk factors

\section{Intra-operative period}

- Anaesthesia workstations should be equipped with means to measure accurate and reliable temperature.

- Ambient temperature in the operating room is recommended to be at least $21^{\circ} \mathrm{C}$ when an adult patient is exposed. The ambient temperature may be reduced after passive and active warming techniques are initiated.

- Passive warming techniques (covering the patient adequately for thermal insulation) should be carried out throughout the intra-operative course.

- The temperature of the patient under general anaesthesia and/or neuraxial anaesthesia should be taken and documented at intervals that commensurate with the duration and extent of surgery. A recommendation would be every 30 minutes until the end of surgery.

- Active warming is recommended to be undertaken intra-operatively in patients with multiple known risk factors for inadvertent peri-operative hypothermia, and for surgeries lasting $>60$ minutes.

- All intravenous fluids including blood and blood products should be warmed to $\geq 37^{\circ} \mathrm{C}$ (especially when administered $>1000 \mathrm{~mL} /$ hour). Irrigation fluids used by the surgical team is recommended to be warmed to a temperature of $38-40^{\circ} \mathrm{C}$.

\section{Post-operative period}

- The temperature of the patient should be taken and documented on arrival to the postanaesthesia care unit once the patient is stabilised and essential hand-overs are undertaken. Should the patient's temperature be $<36^{\circ} \mathrm{C}$ in the postanaesthesia care unit, temperature monitoring is recommended to be repeated at 15 -minute intervals until normothermia is achieved.

- Patients whose temperature is $<36^{\circ} \mathrm{C}$ is recommended to be actively warmed. It is recommended that the patient should not be discharged from the post-operative anaesthesia care unit until the patient's temperature is $>36^{\circ} \mathrm{C}$.

- Shivering is a physiological response to hypothermia, and should be treated with passive and active warming techniques. Currently there are no known drugs known to prevent and treat shivering, but intravenous pethidine $(12.5-25 \mathrm{mg})$ or dexmedetomidine $(0.5 \mathrm{mcg} / \mathrm{kg}$ over $3-5 \mathrm{~min}) \mathrm{may}$ be considered, although these uses are off-label. ${ }^{11}$

longer surgical duration (estimated at moderate to long) an indication to warm patients and monitor temperatures. Estimated moderate to severe blood loss was also found to be a consideration for temperature measurement and/ or warming by $77 \%$ of participants. More than $80 \%$ of anaesthesiologists would warm their patients actively during the operation if they were $>65$ years old.

Singapore anaesthesiologists considered surgical exposure, surgical duration, patient age and estimated blood loss as risk factors. Based on the survey results and international guidelines, risk factors for inadvertent peri-operative hypothermia are listed in Table 1.

Warming methods. The process that transfers heat to patients is termed active warming: techniques include resistive heating mattress/blanket and forced-air warming devices. ${ }^{12}$ Forced-air warming blankets work on the principle of convective heating, compared to resistive heating mattresses, which works on conductive heating. These methods may be used in combination. Contraindications to active warming include therapeutic hypothermia and impaired thermoregulatory control. ${ }^{3}$ If core temperature is not attentively monitored, overheating may occur. Thermal burns from warming devices though rare, have been reported. ${ }^{13}$

Passive insulation techniques work by thermal insulation, and include the use of cotton blankets, reflective blankets, surgical drapes and plastic sheets. These may be inferior to active warming. Active warming can maintain core temperature better than passive warming by $0.5-1{ }^{\circ} \mathrm{C} .{ }^{14}$

Pre-operative period. Active pre-warming in the pre-operative period using forced-air warming blankets 
and resistive mattresses have been shown to reduce core and peripheral skin temperature gradient and increase the total body heat content, mitigating redistribution of heat post anaesthesia induction. ${ }^{15} \mathrm{~A}$ Cochrane review suggested that active pre-operative warming may be more beneficial than warming only in the intraoperative period. ${ }^{16}$

Sixty-seven percent of anaesthesiologists in Singapore monitored patients' temperatures pre-operatively at various locations. Of these, $81 \%$ measured tympanic temperatures. Only one-third of anaesthesiologists perform pre-warming with most for $<20$ minutes. Seventeen percent did not believe that pre-warming was necessary and $38 \%$ felt there was insufficient pre-warming time. Based on meta-analysis evidence, ${ }^{16}$ our pre-operative recommendations are suggested in Table 1.

Intra-operative period. During the first half hour post anaesthesia induction, the patient's temperature may decrease $>2{ }^{\circ} \mathrm{C}$ due to a combination of impaired thermoregulatory heat-preserving mechanisms, vasodilatation from anaesthesia drugs and procedures with consequential redistribution of heat to the peripheries, and loss of behavioural response to cold. Heat loss from the patient continues due to exposure to the cold operating theatre environment and reduction of metabolic heat production.

A Cochrane review found that intra-operative active warming methods were effective in preventing inadvertent intra-operative hypothermia. ${ }^{16}$ Lower ambient temperatures, administration of cold blood products and/or infusion fluids increase the risk of hypothermia. Appropriate equipment (e.g. in-line warmers) for the warming of blood product and/or infusion fluids should be available in the operating theatre. Irrigation fluids used by the surgeons should be warmed in thermostatically controlled cabinets.

From our survey, most patients' temperatures are monitored continuously (75\%). Passive warming methods, i.e. blankets are available in all practising areas. Forced air warmers are more commonly used compared to electric heating mats and warming gowns. Underbody water mats were the most common (65\%) in use as a method of conductive warming. Fluid infusion warmers were preferred among those surveyed $(97 \%)$. Convection warming methods $(94 \%)$ and warmed fluids $(77 \%)$ were the 2 most preferred intra-operative methods. Seventy-three percent of anaesthesiologists surveyed stated they used active warming for the majority of their patients. Notably, $11 \%$ felt they were limited by the availability of active warming devices, and $20 \%$ were concerned about the cost.
We recommend several intra-operative measures to detect and prevent inadvertent hypothermia (Table 1).

Post-operative period. The post-operative period encompasses the time after the patient is transferred to the postanaesthesia care unit up to 1 day after the operation. ${ }^{7}$ Vigilance in temperature monitoring and treatment of hypothermia should be continued. Postoperative hypothermia may alter drug metabolism (e.g. neuromuscular blocking agents) and stimulate the sympathetic system, potentially putting patients at risk of cardiorespiratory adverse events in the recovery room. Shivering increases oxygen consumption and may occur in up to $60 \%$ of hypothermic patients after anaesthesia. ${ }^{5}$ Shivering should be avoided as it augments metabolic rate and may provoke myocardial ischaemia. ${ }^{4}$

In Singapore, tympanic membrane temperatures are most commonly measured $(80 \%)$. Passive warming (such as blankets) and convection (i.e. forced-air warmer) remain the predominant modes of postoperative warming. Active warming is the most popular $(92 \%)$ method used by anaesthesiologists in Singapore to treat post-operative shivering, followed by passive warming (78\%) and administration of pethidine (50\%). Recommendations for the postanaesthesia care unit are described in Table 1.

Inadvertent peri-operative hypothermia is a common occurrence and is associated with multi-systemic complications, including cardiac morbidity, surgical site infections and bleeding. The peri-operative measures detailed in this document may be undertaken to prevent, detect and manage hypothermia in adult patients. Healthcare professionals should exercise their own discretion and make decisions for their individual patients based on the specific circumstances.

\section{Acknowledgements \\ In addition to the named authors, the following are the members of the Perioperative Hypothermia Prevention Workgroup (Dr Terry Pan, Dr Terence Quah, Dr Tan Soo Guan, Dr Patrick Wong, Dr Teo Li Ming, Dr Mah Chou Liang, Dr Mukesh Shah, Dr Lee Shu Ying, Dr Cheng Yiling, Dr Beatrice Lim, Dr Vera Lim and Dr Bong Choon Looi).}

\section{REFERENCES}

1. Sund-Levander M, Forsberg C, Wahren LK. Normal oral, rectal, tympanic and axillary body temperature in adult men and women: a systematic literature review. Scand J Caring Sci 2002 16:122-8

2. Moola S, Lockwood C. Effectiveness of strategies for the management and/or prevention of hypothermia within the adult perioperative environment. Int J Evid Based Healthe 2011;9:337-45.

3. Duff J, Walker K, Edward KL, et al. Effect of a thermal care bundle on the prevention, detection and treatment of perioperative inadvertent hypothermia. J Clin Nurs 2018;27:1239-49. 
4. Sessler DI. Perioperative thermoregulation and heat balance. Lancet 2016;25:387:2655-64.

5. Torossian A, Bräuer A, Höcker J, et al. Preventing inadvertent perioperative hypothermia. Dtsch Arztebl Int 2015;112:166-72.

6. Hooper VD, Chard R, Clifford T, et al. ASPAN's evidence-based clinical practice guideline for the promotion of perioperative normothermia: second edition. J Perianesth Nurs 2010;25:346-65.

7. NICE Guidelines. Hypothermia: prevention and management in adults having surgery. Clinical guideline [CG65]. Published: 23 April 2008. Last updated: 14 December 2016.

8. Forbes SS, Eskicioglu C, Nathens AB, et al. Best Practice in General Surgery Committee, University of Toronto. Evidence-based guidelines for prevention of perioperative hypothermia. J Am Coll Surg 2009;209:492-503.

9. Torossian A. TEMMP (Thermoregulation in Europe Monitoring and Managing Patient Temperature) Study Group. Survey on intraoperative temperature management in Europe. Eur J Anaesthesiol 2007;24:668-75.

10. Niven DJ, Gaudet JE, Laupland KB, et al. Accuracy of peripheral thermometers for estimating temperature: a systematic review and meta-analysis. Ann Intern Med 2015;17:163:768-77.

11. Liu ZX, Xu FY, Liang X, et al. Efficacy of dexmedetomidine on postoperative shivering: a meta-analysis of clinical trials. Can J Anaesth 2015;62:816-29.

12. De Witte JL, Demeyer C, Vandemaele E. Resistive-heating or forced-air warming for the prevention of redistribution hypothermia. Anesth Analg 2010;110:829-33.

13. Dewar DJ, Fraser JF, Choo KL, et al. Thermal injuries in three children caused by an electrical warming mattress. Br J Anaesth 2004;93:586-9.

14. Alderson P, Campbell G, Smith AF, et al. Thermal insulation for preventing inadvertent perioperative hypothermia. Cochrane Database Syst Rev 2014;6:CD009908.
15. Roberson MC, Dieckmann LS, Rodriguez RE, et al. A review of the evidence for active preoperative warming of adults undergoing general anesthesia. AANA J 2013;81:351-6.

16. Madrid E, Urrútia G, Roqué i Figuls M, et al. Active body surface warming systems for preventing complications caused by inadvertent perioperative hypothermia in adults. Cochrane Database Syst Rev 2016;4:CD009016.

Edwin Chuen Ping Seet, ${ }^{1,2}$ MMED (Anaes),

Edith Ci-Hui Wong, ${ }^{3}$ MBBS,

Sophia Tsong Huey Chew, ${ }^{4,5}$ FANZCA,

Lian Kah $\underline{\mathrm{Ti}},{ }^{2,4}{ }_{\text {MMED (Anaes), }}$

Chandra Mohan $\underline{\text { Kumar, }}{ }^{1,6}{ }_{\text {FRCA (London), }}$

Nelson Ping Ping Chua, ${ }^{7}$ MMED (Anaes)

${ }^{1}$ Department of Anaesthesia, Khoo Teck Puat Hospital, Singapore

${ }^{2}$ Yong Loo Lin School of Medicine, National University of Singapore, Singapore

${ }^{3}$ Ministry of Health Holdings, Singapore

${ }^{4}$ Department of Anaesthesiology, Singapore General Hospital, Singapore

${ }^{5}$ National Heart Centre, Singapore

${ }^{4}$ Department of Anaesthesia, National University Hospital, Singapore

${ }^{6}$ Newcastle University Medical School, Johor Bahru, Malaysia

${ }^{7}$ Department of Anaesthesiology, Tan Tock Seng Hospital, Singapore

Correspondence: A/Prof Edwin Seet, Department of Anaesthesia, Khoo Teck Puat Hospital, 90 Yishun Central, Singapore 768828.

Email: seet.edwin.cp@ktph.com.sg 